\title{
Durability performance assessment of non-standard cementitious materials for buildings: a general method applied to the French context
}

\author{
Pierre Pimienta ${ }^{\mathrm{a}}$, Blandine Albert ${ }^{\mathrm{b}}$, Bruno Huet $^{\mathrm{b}^{*}}$, Michael Dierkens ${ }^{c}$, Philippe Francisco ${ }^{\mathrm{d}}$, \\ Patrick Rougeau ${ }^{\mathrm{d}}$ \\ a Université Paris-Est, CSTB, 84 avenue Jean Jaurès Champs-sur-Marne, 77447 Marne-la-Vallée Cedex 2, France \\ ${ }^{\mathrm{b}}$ LafargeHolcim R\&D, 95 rue du Montmurier, 38291 Saint-Quentin-Fallavier Cedex, France \\ ${ }^{c}$ CEREMA, 25, avenue François Mitterrand - CS 92 803, 69674 Bron Cedex, France \\ ${ }^{d}$ CERIB, 1 Rue des Longs Reages, 28230 Épernon, France
}

\begin{abstract}
This letter focuses on the technical assessment of new products or systems in the Building sector before being placed on the market in the French and European context. In particular, it discusses the assessment of the durability aspect of innovative products or systems based on cementitious materials that do not meet specifications of standards. After a brief review of the regulatory context and existing assessment procedures, an example of method of assessment is proposed. There are six successive stages, from describing an invention to choosing relevant testing methods and assessment criteria. Thematic fact sheets describing the different kinds of known alteration for cementitious materials and associated performance assessment methods are presented. An applicant and an assessor are invited to expand the scope of their investigation in case of significant technological leap forward that involve the use of a product/system in a field of application that may not be covered by the current fact sheets.
\end{abstract}

Keywords: Assessment; Cementitious materials; Durability; Testing; Non-standard

\section{Introduction}

\subsection{Innovation in the building sector}

The building sector is dynamic and involves various actors who are constantly innovating to meet the challenges of their time and to anticipate those to come in the future. Since the large post-war construction projects until recently, these innovations were mostly focused on improving architectural quality, reducing cost, improving worker safety, etc. The new challenges for which the sector is mobilized are the climate change, the economy of natural resources, and user health and comfort. In these areas, recent advances have reduced the overall environmental impact of buildings (reduction of the carbon footprint of materials and of construction and renovation processes, improving building energy performance, installing alternative non-fossil fuel heating systems, etc.). User health and safety are a constant concern, such as volatile organic compound assessment.
Finally, the use of new technologies makes day-to-day life easier with more connected, more "intelligent" buildings.

Research and development has put in a lot of effort and the accelerating rate of innovation in many areas of construction is creating a large number of new building materials and systems. Furthermore, expectations are high and these innovations must be brought to market quickly.

In many cases, these new products and systems comply with the specifications of existing standards. However, when there is a large technological leap forward, standards, structural calculation methods, and current reference texts dealing with their implementation may not always cover the use of product or the system in the targeted field of application.

The drafting of new standards or the adaptation of existing ones to these new products is one possible way forward, but this often involves timelines that are not compatible with the need to place products on the market rapidly. Therefore, in order to speed the arrival of non-standardised products on

\footnotetext{
* Corresponding author: Bruno Huet, LafargeHolcim R\&D, 95 rue du Montmurier, 38291 Saint-Quentin-Fallavier Cedex, France, bruno.m.huet@lafargeholcim.com
} 
the market, industrial players may appeal to voluntary assessment procedures on the national (e.g. "Avis Technique" in France) or European levels (European Technical Assessment accompanied by reference materials for the dimension and execution of appropriate works). This specific technical assessment work also contributes over the long term to the creation and revision of standards.

\subsection{Problem of insurability}

Insurance companies analyse and insure structures based on the risks they may pose to user safety as well as their durability given the construction processes, innovative or not, used to create them.

The texts used as a basis for this analysis are of a regulatory nature and should be imposed on all actors, for some, or are formal texts arising from expert consensus, for others.

The products and processes that fall under the specifications in these texts are considered to be common techniques, and therefore pose a normal risk. They should not be limited in their insurability as long as they are approved by the insurance companies as fit for use within the field of application.

Other products and processes fall within non-conventional techniques and are therefore subject to a specific procedure by insurance companies for the establishment of insurance contracts in line with the French law of 4 January 1978, commonly called the "Spinetta law".

\subsection{Durability assessment details}

While the assessment process may be perceived as complex, any durability assessment methodology such as the one proposed should offer a clear vision of the existing possibilities. It should cover new cementitious materials (new concretes, new concrete products, new constituents, etc.). Because not every topic can be addressed, this letter provides clarifying information specifically about durability assessment.

The example showcased here was launched by Lafarge (today LafargeHolcim). This work is the outcome of a French working group, which included CSTB, in charge of "Assessment" and Lafarge interested in "Technological innovation" with the support of CERIB and CEREMA, especially for their expertise in assessing durability of target materials and for reference materials to normative work.

\section{General process}

A durability assessment methodology should guide an applicant and an assessor as they begin to assess the durability of new cementitious materials. The various possible cases should first be reviewed to assess whether these products fall within a normative framework or not.
In particular, the methodology should be designed for industrial and assessment bodies. It should aim to provide technical elements that will:

- assist in the identification the risks to which these products may be exposed

- $\quad$ help define the consequences of these risks for future material properties

- help select or develop assessment methods to quantify the consequences of the risks specific to each new product.

The standards that the applicant may refer to are listed in section 3. The various national and European assessment procedures are described in section 0 . In terms of durability assessment methodology, there is no single assessment solution because of the multiplicity of aspects of new products or systems within the building sector. When an assessment procedure begins, part of the work done by the organisations in charge of the assessment is to build the assessment framework to be used before marketing the product. For example, it may be necessary to adapt testing methods to the material being assessed and to its planned field of application.

Applicants should have as clear an idea as possible of how their invention will be assessed. This helps to optimise discussions between the applicants and assessment body. It is also important to inform a potential applicant of how they can test their invention starting in the development phase before any formal assessment request, which may result in application failure if the assessment method has not been properly adapted. The proposed method (see section 4) may also apply to solutions that are technological breakthroughs as well as to other more traditional products. Potential applicants are advised to contact an assessment body as early as possible.

The process showcased here relies on thematic fact sheets (see online supplementary material) that address different kinds of alterations as well as the main tests currently used to assess the gravity of their consequences. Using these fact sheets, the different parties will be able to create their own assessment strategy, chose among proposed tests, decide to use a variant, or even design new tests that are as relevant as possible to the properties to be assessed.

\section{The current regulatory context (October 2015)}

This document was written to reflect the current documents published on concrete and cement product durability in a regulatory context.

The standardisation context for concrete structural applications is briefly summarised in Fig. 1 . Other representations of this context are available in standards EN 206 [1] and EN 13670 [2]. The construction of concrete building structures relies on a coherent set of texts that cover the requirements that must be met, product performance, product manufacturing conditions, and integration into the structure, as well as design and process 
factors. For some applications, standards list very precise requirements for the finished product, including durability requirements. For others, standards refer generally to texts that contain shared requirements, such as standards EN 206 [1] and EN 13369 [3].

For applications that fall under standards EN 206 [1] and EN 13369 [3], creating durability performance depends mostly on composition specifications for each exposure class (additional criteria based on "guides" or "recommendations" may be requested in addition to the specifications documents). In France, the use of performance-based methods is not fully integrated into the existing regulations. As needed, the FD CEN/TR 16563 [4] documentation booklet "Principles of the equivalent durability procedure" provides guiding principles for creating an equivalent durability procedure. The application of this procedure requires a national application document (NAD) that does not currently exist in France. The results of the PERFDUB French national project may become the underlying structure for such a future document. The main goal of PERFDUB is to define a methodology on the national scale to assess concrete durability (and that of concrete structures) using a performance-based approach that includes both the "absolute" and "comparative" methods [5].

\section{Non-conventional product assessment procedures}

Innovative building products and systems sometimes fall outside of the scope of current regulations. In this case, the developer can appeal to technical assessment procedures that will help get products on the market in France or Europe. There are two large families of procedures that can be distinguished: national voluntary procedures and European procedures that are governed by Construction Products Regulation (CPR). Below, we describe the main French procedures, ETPM (Preliminary Material Technical Assessment), ATEx (Experimental Technical agreement), and ATec ("Avis Technique" or technical agreement) and the European procedure, ETA (European Technical Assessment). These procedures include product durability assessment based on the target field of application. They are carried out in France by CSTB for the building sector and by CEREMA for the civil engineering sector.

ETPM applies to non-standard construction materials or semi-products. These materials and semi-products are constituents of finished products or systems that will be used in constructions (ex.: cement for making prefabricated concrete products, glass plates for making windows). The manufacturer of these materials generally does not have a direct relationship with the constructor. The final field of application is generally broad and insufficiently defined.

ETPM are carried out at the request of manufacturer on a voluntary basis. They are not covered by regulations.

The goal of ETPMs is to examine and summarise the most relevant pieces of evidence that a product suits a planned field of application, often with a wide scope. ETPM assess the characteristics of a material or semi-product. They do not assess the suitability for use since the product or procedure and the associated field of application are not clearly defined. The document is drawn up jointly by CSTB and the requester. It has two parts: the technical file prepared by the requester and the technical assessment itself prepared by CSTB. Processes created for the assessed innovative materials or semi-products are presumed to later be subject to Atex procedures and/or Avis Technique. That is why the document is submitted to a Specialised Group ("GS") of the technical agreement Commission, even though it is handled outside of the Avis Technique procedure.

ATEx applies to innovative processes that do not yet have worksite references. It takes place before the "Avis Technique". ATEx is made for limited application to a single construction operation that has already been identified or for a limited field of application for similar structures. The reviewed field is also narrower than the one for the Avis Technique. The procedure timeline is also shorter (two-three months). The "Avis Technique" is the most widely known and widely used technical assessment procedure. It is governed by a decree placing it under the supervision of a specific Commission (CCFAT).

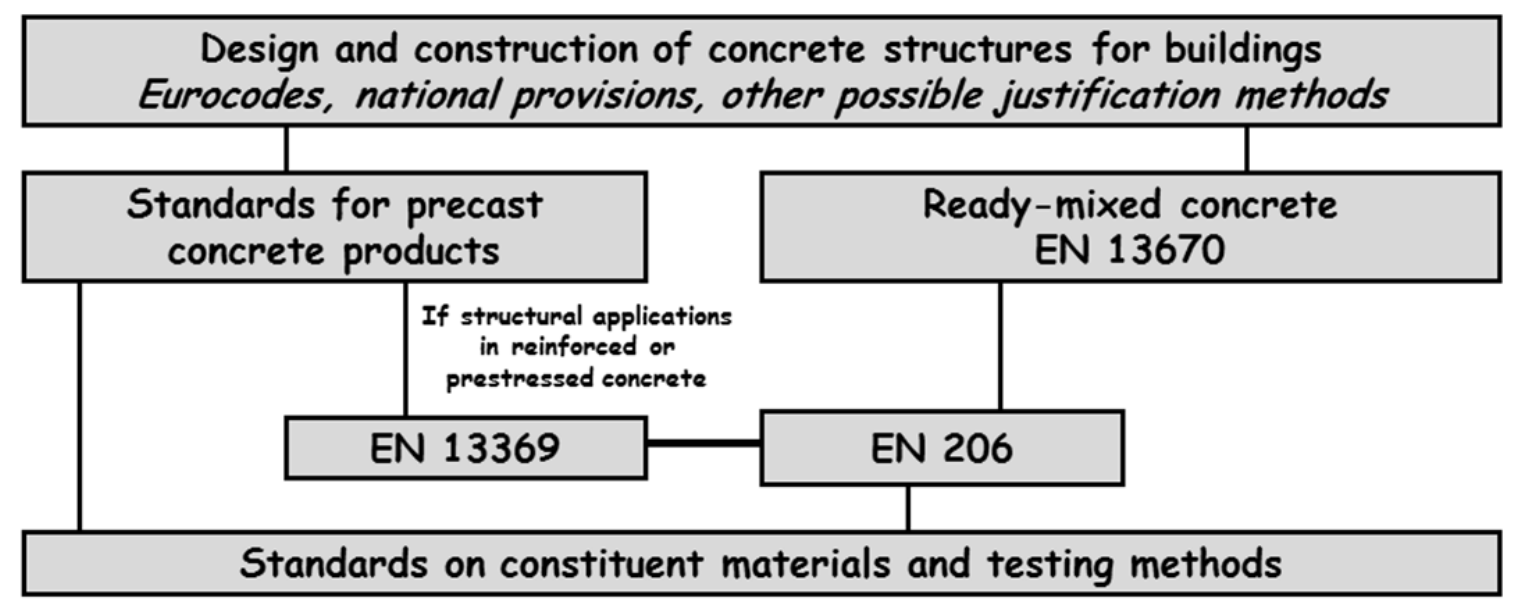

Figure 1. European standardisation context for concrete structures. 
It is meant to provide all construction industry participants with an authorised opinion on new products, processes, and equipment for a specific use. It notably indicates the ways that the process or product:

- complies with current regulations

is suitable for use in construction

provides a known durability in service

These technical agreements are informational documents. They are not mandatory and they do not have any particular regulatory effect. They are created by technical experts working in Specialised Groups brought together by CCFAT.

The agreement has two parts: a technical file summary created by the requester and the opinion itself. It is delivered by CCFAT after examination by Specialised Groups (GS) named by this organisation.

CSTB examines the validity of the evidence in the request file submitted by the requester. The conclusions of this examination are presented to the Specialised Group in charge of creating the agreement. The agreement is then published by CSTB on its website.

The "Avis Technique" applies to new products, procedures, and equipment. Systems and structures whose components, characteristics, and implementation fall under conventional practices are not covered by this procedure.

For products with a CE marking, CCFAT may deliver an agreement in the form of a technical application document (DTA).

European Technical Assessment (ETA) is a European procedure. It is defined in regulation (EU) No. 305/2011 on construction products (CPR), which came fully into force on 1 July 2013. Its key new concept, the Declaration of Performance (DoP), is a new document that replaces the CE compliance declaration following the construction products directive (CPD). Based on the planned use(s) for its product, the manufacturer may chose the product's essential characteristics of which it wishes to declare the performance. Currently, to place construction products on the market that are covered by a harmonised standard, the manufacturer must issue a Declaration of Performance, then apply the CE marking to produces that comply with the Declaration of Performance. For a product that is not (or is not entirely) covered by a harmonised standard, the manufacturer may request an ETE in order to obtain a Declaration of Performance and CE marking. The ETE is issued based on reference to the EAD (European Assessment Document). The EAD is issued jointly by all other relevant European technical assessment organisations before being adopted by EOTA (European Organisation for Technical Assessment: www.eota.eu). When the innovative product is part of a product family that has already received an EAD (quick setting cement, calcium sulfoaluminate cement, etc.) the provisions of this EAD must be taken into account.

The assessment of new products or systems is not fundamentally different from the approach taken in a regulatory context. For example, for a new product, characterisation generally includes composition, properties, and durability characteristics. Assessment methods for innovative products may differ from regulatory methods in cases where, for example, it is necessary to quantify a new risk or a new property that is not covered in an existing standard. It is also sometimes necessary to adapt testing methods due to the particular properties of the proposed innovation. It is then the responsibility of the assessment body to approve the most relevant testing method.

\section{Method for identifying risks and choosing which tests to carry out}

Durability assessment includes several stages. The first two stages include describing the invention (see stage 1) and the properties whose durability is to be assessed for the planned field of application (see stage 2). The next stage involves making a list of the potential kinds of degradation (see stage 3). To do so, a list of the main kinds of degradation known for cementitious materials is proposed in this document. Concise thematic fact sheets describing these degradations are provided as online supplementary material to this document. According to the particular nature of a product or system, other types of degradation may be considered.

The applicant provides evidence that provide a way to qualify the durability over time of the product or system performance. Different means are available, such as lessons learned from the field and laboratory ageing tests (see stage 4). As for laboratory tests, criteria for test method selection and execution are proposed (see stage 5) as well as assessment criteria (stage 6). A method is proposed for cases where a specific method of alteration needs to be taken into account. Finally, the last stage for the assessment organisation is to give an overall assessment of the product's durability by compiling the different data available. It is especially important to take into account the exposure conditions and the lifespan of the structure in which the product will be used.

\subsection{Stage 1: describing the invention}

The goal of this stage is to give a detailed description of the innovation: the product composition, the field of application and the planned lifespan, the possible manufacturing specificities, implementation specificities, and any other information that might be useful to the assessment body.

This stage ensures whether the new product or system proposed is already covered by current standards in force (if so, the durability for the field of application and the planned lifespan are then presumed acquired) or the assessment of durability needs to be carried out.

\subsubsection{The product}

The following questions help to define the product and identify its particular characteristics:

1) How would you describe the product (constituents, mix design, geometry, ...)?

2) Does the product meet a standard?

3) Are the products constituents all standard-compliant or well described in a product standard? 
4) If yes, do the combination and proportions of the constituents fall under a standard?

5) Is the formed cement matrix similar to those formed by conventional standardised binders (hydrate assemblage, porosity, etc.)?

6) Does the product have inclusions (aggregate, fibres) that are not conventional for cementitious materials?

7) Are the properties or characteristics of the final product standardised?

\subsubsection{Field of application}

The field of application is defined by answering the following questions:

- Is the field of application conventional?

- What are the main functions of the product?

- What is its planned lifespan?

- Is it planned to play a structural role?

- If yes, are its mechanical properties compatible with an existing approval method (Eurocodes, etc.) used for structural design?

- Is there a risk of incompatibility between the product and another constituent or material it might come into contact with?

- $\quad$ Are metallic elements used?

- What stresses are foreseen?

- What are the climate characteristics (temperature, relative humidity, freezing, etc.)?

- What are the chemical agents that might be in permanent or occasional contact with the product (natural water, salt, sulphates, acid...)?

- What are the other foreseen stresses (abrasion, permanent or cyclical mechanical loading)?

- Is the product in direct contact with the environment (protective cover)?

\subsubsection{Implementation}

Finally the standard implementation for this product must be described. These items inform the answers to the following questions:

- Are the implementation conditions conventional (like, for example, those specified by DTU 21 [6]) or are they unique?

- Is it necessary to certify product applicators?

\subsection{Stage 2: properties to be verified}

In all cases, the durability of mechanical stability properties must be verified. The applicants can also claim the durability of other properties if they so wish.

N.B.: Harmlessness to people (construction workers, users) and the environment at different stages in the products lifespan are not included in this guide, but must be assessed according to the regulations in force.

\subsection{Stage 3: degradation risks}

The inventory of degradation risks that would lead to significant performance loss must be an exhaustive list based on the product specificities, its use, and its planned environment. Table 1 is a tool to help with this inventory. It lists the known degradations for conventional concrete. It is also important to think of additional risks specific to the invention as needed.

The mechanisms for cementitious material alterations are often complex. These mechanisms are described in the example showcased here in thematic fact sheets (see online supplementary material) in order to provide a guide to understand them and decide if they need to be taken into account. These fact sheets cover the known potential degradation mechanisms for cementitious materials leading to a risk of loss of mechanical integrity. In the case of an alteration not covered by these fact sheets, a specific one may be created using the structure of those presented in this document.

Table 1. List of performance loss risks for the invention.

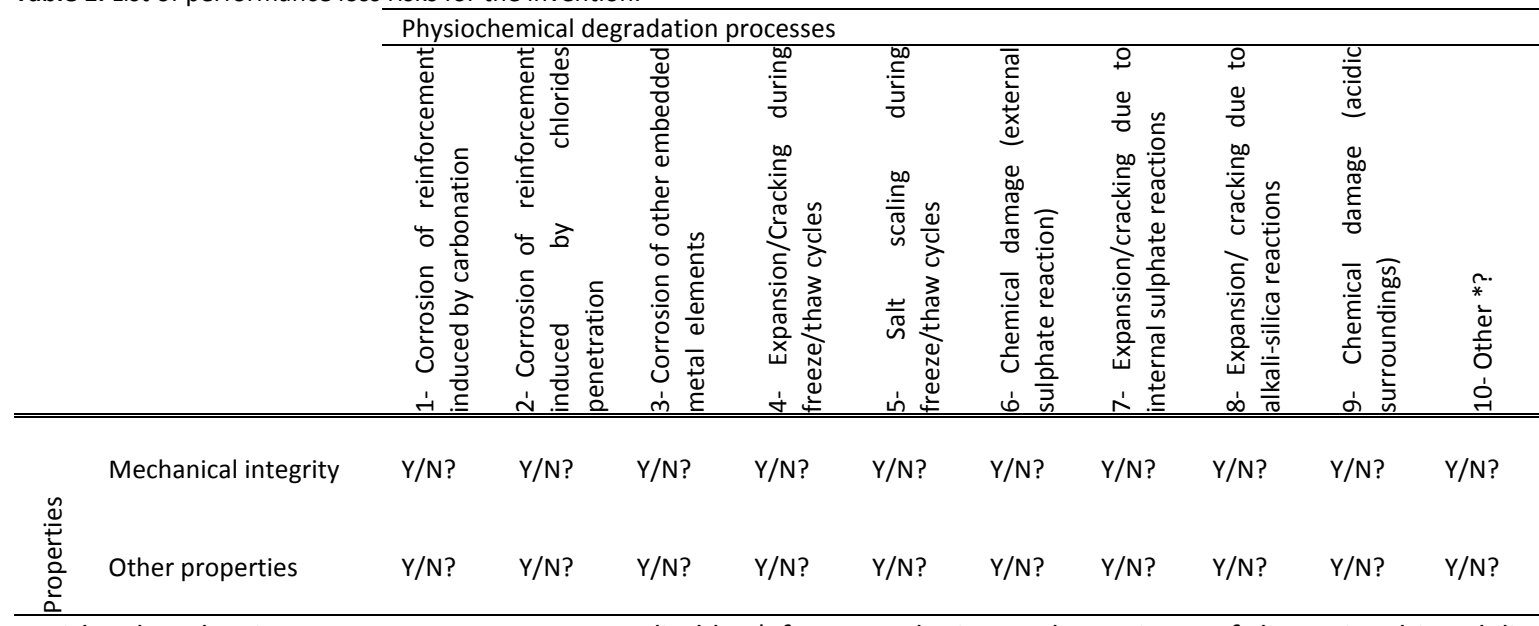

Y: Risk to be taken into account.

$\mathrm{N}$ : Not applicable. ${ }^{*}$ for example, it may be an issue of dimensional instability due to contact with water. 
This approach may also be used to describe changes in other properties. For example, dimensional instability leading to material cracking in its environment might be considered.

The structure of the pathology sheets is as follows:

1) Introduction

2) Consequences

3) Physico-chemical mechanisms

4) Main models

5) Main parameters

6) Testing method stages

7) Reference testing methods

8) Performance assessment

9) References

\subsection{Stage 4: evidence for the assessment}

Assessing the consequences of an identified risk can rely on:

- qualification tests described in section 4.5 below

- lessons learned from the field (existing structures or test specimens)

- results of previous tests (technical documents, publication)

- an analysis of physio-chemical mechanisms (see sections 3 and 4 of the pathology sheets)

- calculation results

- etc.

\subsection{Stage 5: testing methods for each property-degradation mode couple}

An approach for selecting relevant laboratory tests is provided below and illustrated in Fig. 2:

1) The first step involves checking if there is a relevant test in the current provisions in force for the area of use to assess this durability. If that is the case, this test must be preferred. A non-exhaustive list of possible tests is given in the thematic fact sheets; see "reference testing methods" in online supplementary material.

2) If that is not the case, it is possible to adapt a relevant reference test as long as the principle of the test is not undermined. Changes to testing conditions must be minor and may not modify the degradation mechanisms being studied. It is important to consult section 5 "Main parameters" on the fact sheets to determine what testing conditions might be adapted.

3) If neither of the two previous solutions is possible, a test developed and used by a single laboratory may be used, for example. This test should be suitable and credible. Section 7 of the fact sheets also proposes specific and documented tests.

4) Finally, as a last resort, a new test must be proposed. This test may be created using the pathology sheets based on "physio-chemical mechanisms" (section 3) and "main parameters (section 5). This new test must be described exhaustively.

5) Tests must be carried out using the selected method.

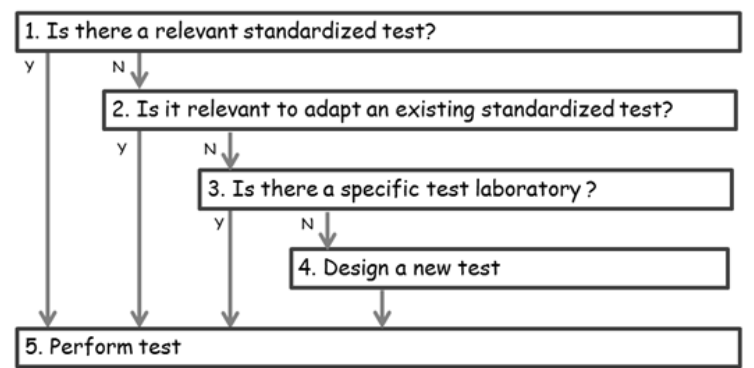

Figure 2. proposed approach for choosing which laboratory tests to carry out

\subsection{Stage 6: assessment method}

The goal of this section is to identify the different available methods for reaching a conclusion about the product or system ability to maintain its properties.

Two main methods are possible [1,4]: "Absolute" approach and "Comparative" approach. The "absolute" approach is based on a comparison between threshold values and measured values using the same testing methods for both measurements to establish a performance level. There are two possible choices:

1) Ideally, it is best to use the threshold values available in the existing provisions in the place of use. Section 7 of the fact sheets lists technical documents. These documents list thresholds in some cases.

2) As a second choice, the threshold values defined in the calculation methods may be used. Section 4 of the fact sheets lists the main models.

The "comparative" approach is based on a comparison between measured values derived from the product and from a reference material. It is important to make the reference material choice explicit. Note that it is advisable to take account of uncertainties of test results.

Finally, it is important to come to a conclusion on the conformity of the invention to the description provided by the applicant as well as to establish whether there are no non-compliances.

\section{Acknowledgements}

The authors would like to thank Michel Delort and Laurent Izoret, chairs of CEN/TC51 (Cement and Construction Lime) standardisation committee, for their contribution to the working group and to the creation of this document.

The authors would also like to thank the following editors for the significant improvements they made to this document: Charles Baloche, CSTB, Francois Boutin, CSTB, Anca Cronopol, CSTB, David Henriques, CSTB, Yannick Lemoigne, CSTB, Nicolas Ruaux, CSTB, Remi Barbarulo, LafargeHolcim R\&D, Eric Brouard, LafargeHolcim R\&D, Francois Delarrard, LafargeHolcim R\&D, Edouard Gengembre, LafargeHolcim R\&D, Gabriel Pham, LafargeHolcim R\&D, François Jacquemot, CERIB, Jonathan Mai-Nhu, CERIB, Marjorie Petitpain, CERIB 


\section{References}

[1] NF EN 206:2014. Concrete - Specification, performance, production and conformity, 2014.

[2] NF EN 13670:2013 Execution of concrete structures, 2013.

[3] NF EN 13369:2013. Common rules for precast concrete products, 2013.

[4] FD CEN/TR 16563:2014. Principles Of The Equivalent Durability Procedure, 2014.

[5] Irex, The National Research Project PERFDUB, 2015, http://www.perfdub.fr/en/.

[6] NF P18-201:2004. DTU 21 - Building works - Execution of concrete works - Technical specifications, 2004. 